Cómo citar este artículo: López-Posada, J. C., \& Pachón-Ariza, F. A. (2017). Identificación de ventajas y desventajas de los canales de comercialización en las economías campesinas de dos municipios de Meta y Cundinamarca, Colombia. Rev.investig.desarro.innov, 8(1), 35-47.

\title{
Identificación de ventajas y desventajas de los canales de comercialización en las economías campesinas de dos municipios de Meta y Cundinamarca, Colombia
}

\section{Identification of advantages and disadvantages of commercialisation channels in peasant economies in two municipalities from Meta and Cundinamarca, Colombia}

\author{
Juan Carlos López-Posada' \\ Fabio Alberto Pachón-Ariza ${ }^{2}$
}

Recibido: abril 24 de 2017

Aceptado: junio 29 de 2017

\section{Resumen}

Una de las principales funciones de la economía campesina es la producción de alimentos para autoconsumo. Esta actividad es realizada sin desmedro de la generación de excedentes para comercializar y así satisfacer otras necesidades básicas; de esta forma las familias campesinas organizan su producción. En este contexto, los campesinos buscan diferentes canales para comercializar sus productos. Para los casos estudiados, municipios de Fuente de Oro, departamento del Meta; y El Colegio, departamento de Cundinamarca, la comercialización se hace a través del Programa Mercados Campesinos, pero también por medio de otros canales fuera del Programa. Mediante la metodología 'Circuitos de Mercados Campesinos' se hizo acompañamiento a una familia de cada municipio, con el fin de identificar ventajas y desventajas de los canales de comercialización usados por ellos. Los hallazgos muestran que la comercialización a través de los Mercados Campesinos es la preferida, aún cuando se continúa en la búsqueda de canales propios para comercializar. La limitante más relevante que se identificó durante el proceso es el transporte, lo cual hace que el campesinado recurra a la intermediación.

Palabras clave: acceso a mercados, alternativas de comercialización de alimentos, comercio justo.

\begin{abstract}
One of the primary functions of the peasant economy is the production of food for self-consumption. This activity is carried out without the detriment of the generation of surpluses to commercialise and thus satisfy other essential needs; in this way, the peasant families organise their production. Given this context, farmers seek for different channels to commercialise their products. For the analysed cases, ( the municipalities of Fuente de Oro, in Meta State, and El Colegio, in Cundinamarca State), the commercialisation of products has been carried out through the program called 'Mercados Campesinos' and other alternative channels. Through the methodology 'Circuito de Mercados Campesinos', an accompaniment to a family from each municipality was made to identify the advantages and disadvantages of the commercialisation channels used by them. The findings show that the marketing in the 'Mercados Campesinos' is the favourite one, even though, they continue searching for alternative channels to access other markets. The most significant limitation that was identified during the process was transporting, which makes that peasants look for the middleman to facilitate getting to markets.
\end{abstract}

Keywords: getting markets, alternatives to food markets, fair trade.

1 Ingeniero Agrónomo, Universidad Nacional de Colombia, Bogotá, Colombia. E-mail: jclopezp@unal.edu.co

2 Médico Veterinario, Dr. rer. agr., Universidad Nacional de Colombia, Bogotá, Colombia. E-mail: fapachona@unal.edu.co 


\section{Introducción}

Colombia cuenta con una ruralidad muy diversa, la cual ha sido inadecuadamente atendida por las políticas públicas durante los últimos gobiernos. Esta realidad ha provocado condiciones que van en desmedro de la economía campesina, reflejada en situaciones como alta concentración de la tierra, desmonte de los sistemas de asistencia técnica e investigación, subsidios tendientes a beneficiar a grandes capitalistas rurales, altos precios de insumos comparado con los países vecinos, escasas oportunidades de una pertinente educación, difícil acceso a sistemas de salud, exigua posibilidad de acceder a una pensión de vejez, entre muchas otras. Lo anterior ha generado procesos de aislamiento y precariedad evidentes en muchas zonas rurales de Colombia.

En relación con los procesos productivos de la economía campesina, el uso de su recurso productivo más importante, la mano de obra familiar, ha sido una característica primordial como estrategia para superar la falta de acceso a tierra y capital. Igualmente, es utilizado como maniobra para superar los efectos negativos derivados de la implementación de las políticas públicas.

La discusión relacionada con economía campesina, su significado e importancia para Latinoamérica ha sido muy nutrida. Un primer acercamiento a esta discusión es aquella que plantea la racionalidad productiva de las familias campesinas en relación con sus condiciones y situación inmediata de producción, de organización, gestión y planificación de actividades. Es caracterizada por el uso de la fuerza de trabajo familiar, así como escasos recursos de tierra y tecnología (Forero, 2000; Forero, 2003; Machado, 2003; Plaza, 1992). También es de destacar que la economía campesina combina la producción para el mercado con la producción para el autoconsumo como la principal característica de esta economía (Benítez, 2009).

Según Gonzalves (2007), la economía campesina es un modo de producción con una lógica inter- na autónoma y propia. Es una unidad de consumo y de producción, y parece mostrar un comportamiento económico motivado por asegurar la auto-subsistencia, que se articula externamente con el capitalismo. Bartra (1982) agrega a la discusión que la economía campesina como unidad socioeconómica, es como una célula de producción y consumo constituida por una unidad orgánica de trabajo y medios de producción, a lo que Bernstein (2010) añade que su funcionamiento y sobrevivencia le ha permitido la reproducción de los hogares rurales.

Chayanov (1986), como uno de los autores más debatidos sobre la propuesta de economía campesina, indica que el trabajo no está separado de las formas de organización, y sustenta en tres puntos la teoría del trabajo campesino. Primero, debe existir la autosatisfacción del campesino por su trabajo, el cual no es obligado; segundo, la organización dada alrededor de la economía campesina no tiene fines capitalistas, sin embargo están relacionadas. Por último, para la satisfacción de las necesidades de la comunidad se organizarán en el trabajo. En este orden de ideas el trabajo debe obedecer no a las formas productivistas de acumulación de capital, ni mucho menos a formas de esclavización del hombre, el fruto de dicho trabajo debe responder a la satisfacción de las necesidades de su familia y comunidad (Pitre-Redondo, Cardona-Arbeláez, \& Hernández-Palma, 2017).

La economía campesina descansa básicamente en la combinación equilibrada entre los bienes generados para el mercado y los generados para el consumo de la familia a partir del trabajo desarrollado en la propia finca y con predominio de la mano de obra familiar (Benítez, 2009; Sierra-Roberto, 2012). Estos principios, al igual que los de generación de ingresos en procesos de comercialización más justos, son promovidos por el Programa Mercados Campesinos, PMC, que ha sido implementado en los últimos años, especialmente en la región central de Colombia. 
El Programa de Mercados Campesinos, PMC, es un esfuerzo que busca generar organización campesina, en este caso en función de una justa comercialización de sus productos y así propender por la satisfacción de las necesidades básicas de los campesinos. Desde el año 2004 el Programa toma mayor fuerza, con el objetivo de construir nuevas alternativas de comercialización que beneficien tanto al productor como al consumidor. Este proceso da cuenta de la participación de más de 2.400 familias de productores campesinos, en 65 municipios de los departamentos de Boyacá, Cundinamarca, Meta y Tolima. Con una fuerte operación logística y comercial, ofrecen sus productos en plazas y parques de Bogotá. PMC, promovido por varias organizaciones, busca consolidar dos pilares. Primero, se trabaja por la consolidación de políticas públicas de Soberanía Alimentaria y la superación de la población rural. Y segundo, busca la consolidación de una economía campesina desde la producción, transformación y comercialización de productos, además de la preocupación por entregar al consumidor un producto de calidad, inocuo y a un precio justo (ILSA, Campesina, \& OXFAM, 2010).

PMC promueve la comercialización de productos agrícolas bajo producción convencional y orgánica, en fresco y procesados, ofreciendo una mejor calidad para el consumidor, y permitiendo un ahorro en la transacción de hasta un 30\% (Parrado, \& Molina, 2014). Se rige por el acuerdo 455 de 2010 del Concejo de Bogotá por el cual se promueve la integración regional rural a través de mercados temporales campesinos, indígenas, afrodescendientes y demás etnias, de manera tal que finalmente se forman los llamados mercados presenciales (Parrado, \& Molina, 2014). Los mercados presenciales son unos espacios en donde se encuentran de una manera informal, aunque institucionalizada, productor y consumidor, dándose un diálogo directo entre las partes, estableciéndose relaciones de confianza, y como resultado de ello ofreciendo un valor agregado a quien vende, evidenciado en una valoración adecuada por parte del consumidor. Así mismo, la certeza de que quien está vendiendo el producto es el mismo productor, y por tanto, hay una directa remuneración de su producto. Además, con una disminución del precio para el consumidor (Chaparro, 2014).

PMC cuenta con una estructura administrativa por áreas de trabajo en lo organizacional, productivo, mercadeo, comunicaciones, formación y educación. Todo esto apoyado por una dirección política y técnica. Sin embargo, el trabajo en lo rural es realizado por la base política, que son los mismos campesinos, organizados bajo los Comités Campesinos Comunales. Dicha base conforma el Comité de Interlocución Campesino y Comunal (CICC), del cual hacen parte organizaciones campesinas con interés de forjar una nueva sociedad desde el sector rural. Dichas organizaciones son: Asociación Campesina Colombiana (ACC), Asociación Nacional de Ayuda Solidaria (ANDAS), Asociación Departamental Campesinos de Cundinamarca (ADUC), Asociación Nacional de Mujeres Campesinas e Indígenas de Colombia (ANMUCIC), Federación Nacional de Cooperativas Agrarias (FENACOA), Federación Nacional Sindical Agropecuaria (FENSUAGRO), Confederación Nacional de Acción Comunitaria (CONFECOMUNAL) y Fundación San Isidro (FSI) (ILSA et al., 2010).

Tomando como base lo anterior, este artículo busca responder a la siguiente pregunta: ¿Cuáles son las ventajas y desventajas para los productores campesinos de Fuente de Oro y El Colegio de participar en el Programa Mercados Campesinos?.

\section{Metodología}

Para responder el interrogante planteado en este artículo, se identificaron los canales de comercialización tanto en los mercados presenciales como los canales tradicionales que tienen los productores campesinos de Fuente de Oro y El Colegio, municipios de Meta y Cundinamarca, respectivamente, buscando evaluar las ventajas y desventajas que cada uno de ellos tiene y así establecer si los otros canales son mejores que aquellos que encuentran en el PMC. 
La metodología utilizada se planteó en tres fases. En la primera fase se identificaron los circuitos de los mercados campesinos, como metodología propia del Grupo de Investigación en Gestión y Desarrollo Rural (Parrado, \& Gutierrez, 2014). A esta metodología se le realizó una adaptación para un segundo tipo de circuitos denominado, circuitos de mercados de autogestión (MA). En la segunda fase se identificaron las variables y ejes de análisis que sirvieron para comparar los dos circuitos de mercado. Finalmente, la última fase consistió en caracterizar y comparar los canales de comercialización identificados durante la aplicación de la metodología utilizada.

\subsection{Ubicación de la investigación}

El municipio de Fuente de Oro, está ubicado en el departamento del Meta a orillas del rio Ariari. Situado en una altitud de 359 msnm, su clima es cálido y su área rural se encuentra dividida en 32 veredas. La investigación se realizó en la vereda Alto de Sardinata, en las fincas Miramar y Primavera en un total de 4 ha. El municipio de El colegio está ubicado en el departamento de Cundinamarca, con una altitud de 997 msnm, el área rural está dividida en 41 veredas aproximadamente. Para este caso la investigación se llevó a cabo en la vereda Honduras, en dos fincas que suman 11 ha.

\subsection{Circuitos de los mercados campesinos}

Los circuitos de los mercados campesinos como metodología se apoya en las herramientas de trabajo observación participante y entrevista semi-estructurada. Estas herramientas se usaron para realizar la caracterización de los circuitos con las familias productoras. Esta fase se compone de cuatro escenarios (Figura 1). Estos escenarios hacen referencia a la logística realizada por los productores desde su predio hasta llegar al acopio municipal. El primer escenario identifica los procesos llevados a cabo en la finca de la familia campesina. El segundo escenario identifica aspectos del acopio municipal y el transporte hasta la llegada a la ciudad de Bogotá. Esta fase es coordinada por cada comité municipal. El tercer escenario busca caracterizar la organización en la llegada a Bogotá, además la distribución y criterios de ubicación en los mercados presenciales. El cuarto escenario propuesto es identificar el regreso de los productores al predio (Parrado, \& Gutiérrez, 2014).

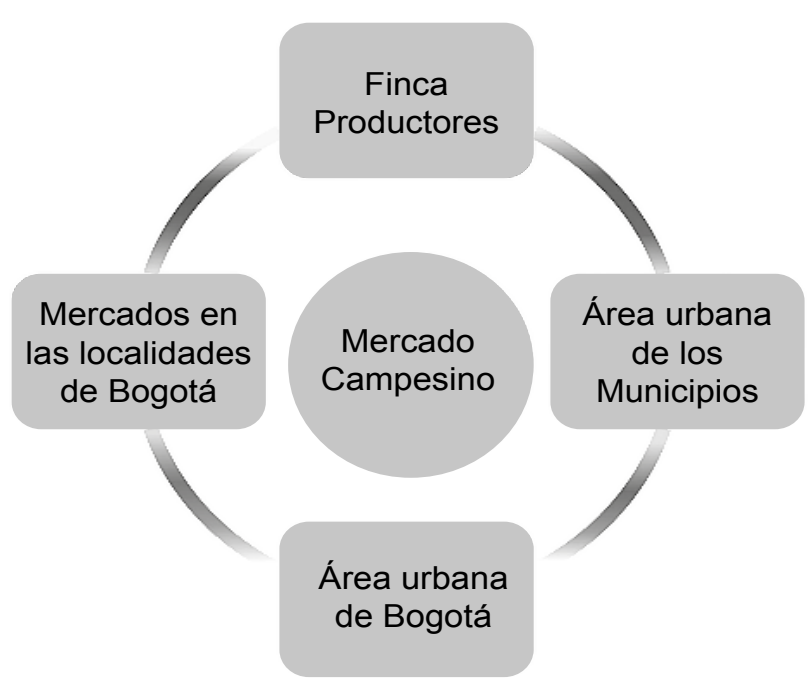

Figura 1. Circuito de los Mercados Campesinos.

Para analizar los circuitos de los Mercados de Autogestión, MA, se realizó un ajuste a la anterior metodología; este se refleja en la logística que debe realizar el productor campesino para vender sus productos por fuera del programa MC.

\subsection{Variables y ejes de análisis}

La información recolectada se evaluó tomando en cuenta las variables y sus respectivos ejes de análisis, listados en la tabla 1. 


\begin{tabular}{|c|c|c|}
\hline Variable & Eje de análisis & Unidades de medición \\
\hline \multirow[t]{2}{*}{$\begin{array}{l}\text { Preparación (Cosecha y Posco- } \\
\text { secha) }\end{array}$} & $\begin{array}{r}\text { Calidad y cantidad del producto } \\
\text { Utilidad neta }\end{array}$ & $\begin{array}{l}\text { Kilogramos de producto de } \\
\text { primera, segunda o tercera calidad }\end{array}$ \\
\hline & $\begin{array}{r}\text { Tiempo } \\
\text { Grado de satisfacción }\end{array}$ & $\begin{array}{l}\text { Ganancias luego de restar los ingresos menos } \\
\text { egresos }\end{array}$ \\
\hline Transporte & & $\begin{array}{l}\text { Horas dedicadas a diferentes actividades desde } \\
\text { cosecha hasta la venta }\end{array}$ \\
\hline & & $\begin{array}{l}\text { Escala de } 1 \text { a } 5 \text {, donde } 1 \text { es insatisfecho y } 5 \text { sa- } \\
\text { tisfecho }\end{array}$ \\
\hline Logística inversa & & \\
\hline
\end{tabular}

Tabla 1. Variables y ejes de análisis.

2.4 Canales de comercialización

La Tabla 2 muestra la descripción de los canales y sus respectivas vías de comercialización.

\begin{tabular}{|c|c|c|}
\hline Canal de comercialización & Vía de comercialización & Descripción \\
\hline \multirow[b]{2}{*}{ Mercados campesinos } & Mercados Presenciales & $\begin{array}{l}\text { Se refieren a la comercialización en los parques estableci- } \\
\text { dos por el programa Mercados Campesinos. Cada } 15 \text { días } \\
\text { en la ciudad de Bogotá. La comercialización es directa } \\
\text { productor-consumidor. }\end{array}$ \\
\hline & Mercados Mayoristas & $\begin{array}{l}\text { Son los lugares donde se hacen las ventas por medio de } \\
\text { comité de comercialización del PMC: fruver, plazas de } \\
\text { mercado, tiendas de barrio, mercados institucionales. En } \\
\text { la mayoría de los casos, quien hace la negociación es el } \\
\text { mismo productor. }\end{array}$ \\
\hline \multirow{2}{*}{ Mercados Tradicionales } & $\begin{array}{l}\text { Mercados Intermedia- } \\
\text { rios }\end{array}$ & $\begin{array}{l}\text { Son las ventas que se hacen al intermediario de la muni- } \\
\text { cipalidad, en la puerta del predio. }\end{array}$ \\
\hline & Mercados Autogestión & $\begin{array}{l}\text { Estos son las ventas que se hacen sin el apoyo del PMC, es } \\
\text { decir por acción propia del productor. }\end{array}$ \\
\hline
\end{tabular}

Tabla 2. Resumen canales y vías de comercialización en los municipios de Fuente de Oro y El Colegio.

\subsection{Recolección de datos}

Los datos cuantitativos y cualitativos fueron colectados mediante observación participante durante todos los procesos de los circuitos de mercado y de autogestión, lo cual incluye desde la preparación para la cosecha, hasta la venta final del producto. En algunos casos, la observación podía tomar entre 5 y 7 días. En Fuente de oro, los circuitos se realizaron con los agricultores de las fincas Primavera y Miramar. En el Colegio, el acompañamiento en los circuitos se realizó con las fincas Vencedor y San Carlos.

\section{Resultados y discusión}

\subsection{Caso Fuente de Oro}

La producción agropecuaria de los productores de Fuente de Oro está representada en plátano, yuca y limón. Sin embargo, como se mencionó anteriormente, una de las características de las economías 
campesinas es la diversidad, y este caso no es la excepción. Por tanto también producen zapote, guanábana, mango, naranja, cacao, aguacate, noni. Además, en el aspecto pecuario la producción se centra en porcinos, aves, bovinos y peces.

Luego de la participación en los dos Circuitos de Mercado, se evidenció que las familias utilizan ambos canales de comercialización: Mercados Cam- pesinos y Mercados Tradicionales, como se describirá con profundidad más adelante. Como vías de comercialización, se identificaron los Mercados Presenciales, Mercados Mayoristas y Mercados de Autogestión. No utilizan la intermediación, aún cuando la zona se caracteriza por presentar un gran número de intermediarios, las cuales serán descritas posteriormente. Ellos tienen logísticas similares en cada vía. Sin embargo, se pueden identificar algunas diferencias. (Figura 2)

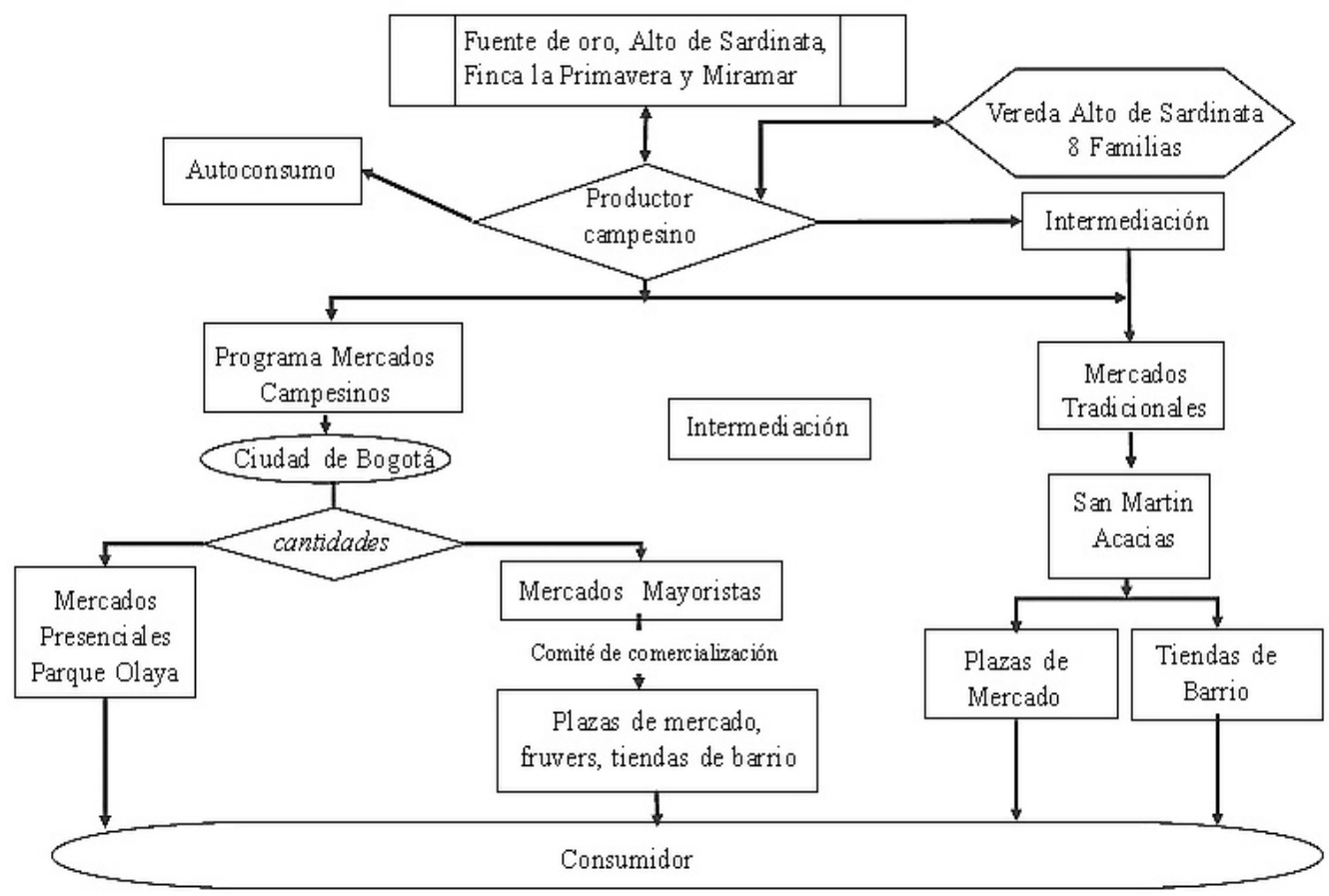

Figura 2. Diagrama de flujo caso Fuente de Oro.

\section{Preparación}

En la fase de preparación se verificó la logística desde el predio del productor con el fin de observar la manera como preparan el producto para la venta, por ello es importante mencionar algunos aspectos relevantes realizados durante la cosecha y poscosecha. Para estos procesos los pequeños productores no cuentan con tecnologías avanzadas, lo que implica que deben ser realizados con pocos días de anterioridad al mercado. En el caso de la cosecha, se comienza a colectar aguacate, na- ranjas, limones tres días antes del Mercado Presencial que es el sábado. Dos días antes se cosecha la yuca y el plátano. En la poscosecha, dependiendo la vía de comercialización el empaque es diferente. Para Mercados Campesinos en sus dos vías de comercialización, el plátano es empacado en bolsas de $20 \mathrm{~kg}$ diferenciando tres calidades. Para el Mercado de Autogestión, la venta es en el mismo racimo, pero por arrobas. De igual forma se realiza con la yuca, pero en bolsas de $30 \mathrm{~kg}$. El limón se empaca en bultos para Mercados Campesinos y en canastillas para Mercados de Autogestión. En 
cuanto a costos, el productor contrata un jornal de $\$ 25.000$, el empaque cuesta $\$ 200$ si es bolsa y $\$ 1000$ en el caso de costal.

\section{Transporte}

El transporte para Bogotá en el caso de Mercados Campesinos se realiza mediante un camión con una capacidad de 3 ton. El trayecto dura 8 horas aproximadamente, desde la puerta del predio hasta un punto determinado en Bogotá. Allí el productor debe asumir un costo adicional por traslado dentro de la ciudad. Sin embargo, con ayuda del Comité de Comercialización, este costo se puede descartar o disminuir. Para el Mercado Tradicional el transporte se realiza en la camioneta de propiedad de los productores, el cual dura el mismo tiempo. En cuanto a costos de transporte, son similares para Bogotá o ciudades cercanas, porque se requiere un alto gasto de combustible para realizar la comercialización allí. El costo de dicho transporte está entre \$2000 y \$5000, dependiendo la cantidad de $\mathrm{kg}$ por producto.

\section{Comercialización}

De la totalidad de los productos del caso de Fuente de Oro, $57 \%$ se comercializa en el canal Mercados Campesinos, de los cuales el $18 \%$ se hace por vía Mercados Presenciales, y el 39\% en la vía Mercados Mayoristas. El restante $43 \%$ de los productos se comercializa por el canal Mercados Tradicionales por la vía de Mercados de Autogestión. Sin embargo, los datos que se describirán más adelante muestran un mejor resultado con el plátano y la yuca a través del Mercado Presencial y Mercado Mayorista; mientras que en el limón es más beneficioso a través de los
Mercados de Autogestión. Para los demás productos es indiferente la vía que se use. Dependiendo de la vía de comercialización el productor gasta un determinado tiempo en la venta, siendo en ambos Mercados, Presencial y Autogestión, aproximadamente de 10 a 12 horas, dependiendo de la afluencia de consumidores. Por otro lado, la comercialización de Mercados Mayoristas puede implicar un tiempo muy corto entre 2 a 4 horas. La principal diferencia radica en la forma de pago. Para el caso de Mercados Mayoristas tarda entre 2 y 15 días; mientras que en los Mercados Presencial y Autogestión se recibe el dinero el mismo día.

Aunque los Mercados Presenciales son la vía de comercialización menos utilizada, es donde el productor recibe un mayor ingreso por arroba de producto, en comparación con las otras vías de comercialización, las cuales incluso generan pérdidas para los productores (Tabla 3). Esta ganancia adicional puede alcanzar hasta un $50 \%$ más que en otras vías. Sin embargo, es importante aclarar que el Mercado Presencial requiere de un mayor tiempo y existen costos que el productor no asume. Otro aspecto es que esta es una vía por la cual se comercializan bajas cantidades de producto. Aunque estas cantidades pueden ser suficientes para garantizar la economía del productor, existen excedentes a comercializar que generan ingresos adicionales. Por último, el grado de satisfacción de la familia campesina es mayor en los Mercados Presenciales y de Autogestión, explicado en el hecho de que se recibe el dinero de una manera más rápida. En contraste, los Mercados Mayoristas no son tan satisfactorios para el productor, dado que debe esperar un mayor tiempo para recibir este dinero. 


\begin{tabular}{lccc}
\hline \multicolumn{1}{c}{ Producto } & Mercados Presenciales & $\begin{array}{c}\text { Mercados } \\
\text { Mayoristas }\end{array}$ & $\begin{array}{c}\text { Mercados } \\
\text { Autogestión }\end{array}$ \\
\hline Plátano primera & 4800 & 2300 & 300 \\
Plátano segunda & 2300 & 425 & -700 \\
Plátano tercera & 2300 & -1450 & -1700 \\
Yuca primera & 4800 & -1033 & 300 \\
Yuca segunda & 4800 & -2700 & -1700 \\
Limón única & 11000 & 3500 & 6000 \\
Aguacate única & 17000 & 9500 & 9500 \\
\hline
\end{tabular}

Tabla 3. Utilidad neta en pesos Colombianos por arroba de producto. Caso Fuente de Oro.

\subsection{Caso El Colegio}

Para los productores de El Colegio, Cundinamarca, la producción agropecuaria está representada principalmente en los cultivos de mandarina, na- ranja, banano, plátano, mango y aguacate. Luego del acompañamiento en los dos Circuitos de Mercados, se evidenció que utilizan los dos canales de comercialización: Mercados Campesinos y en Mercados Tradicionales. La Figura 3 muestra los flujos de estos canales.

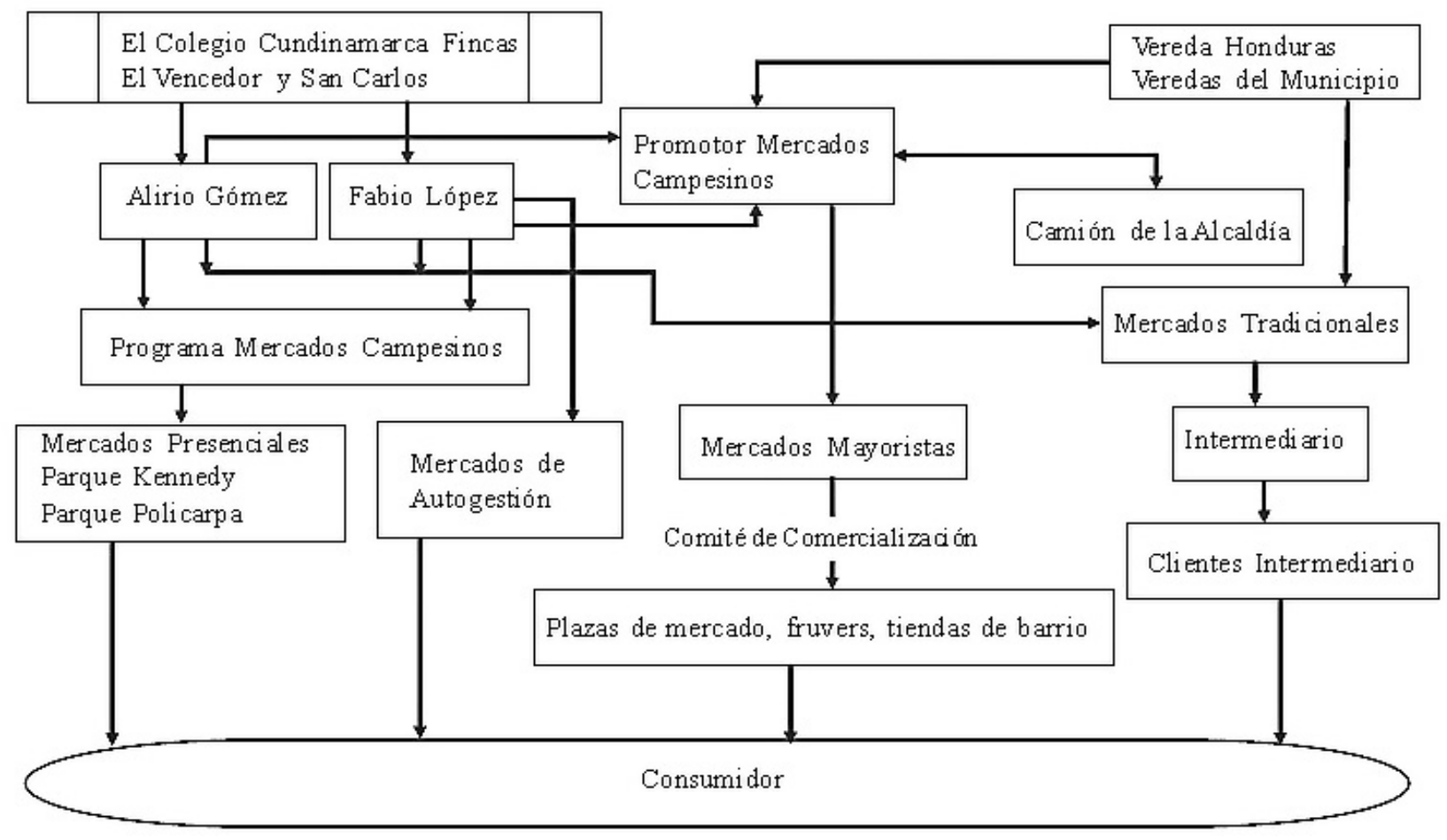

Figura 3. Diagrama caso El Colegio. 


\section{Preparación}

En la fase de preparación se validó la logística necesaria para preparar el producto para la venta en los diferentes canales, teniendo en cuenta cosecha y poscosecha. Al igual que en el caso anterior se inicia a cosechar con ocho días de anticipación al Mercado Presencial con el fin de lograr una adecuada maduración de los plátanos y bananos. Para las mandarinas, naranjas y mangos la cosecha se inicia dos días antes del mercado. El día viernes se ubica el producto a mediodía en las puertas de las fincas, para que el transporte del Programa lo lleve a Bogotá o a los lugares definitivos de comercialización. Así mismo, se preparan para los mercados tradicionales, destacando que los productores de EI Colegio comercializan cada 15 días en los Mercados Presenciales y Mayoristas, y semanalmente en los Mercados Tradicionales. Para ambos casos se realiza empaque en canastillas plásticas.

\section{Transporte}

El recorrido desde las veredas hasta Bogotá demora entre 4 ó 5 horas. La alcaldía municipal facilita un camión en el cual se realiza la recolección en las diferentes veredas, por un valor de tres mil pesos por canastilla (\$3000). El camión hace recorrido los martes y viernes.

\section{Comercialización}

El principal canal de comercialización de los productores con un 75\%, es Mercados Campesinos mediante la vía del Mercado Presencial. Es de destacar que el $75 \%$ de los productos se vende en los parques de Bogotá. También se identificó que bananos y plátanos, en un $70 \%$, se comercializan en Mercados Presenciales y un $30 \%$ por medio de otras vías, como el Mercado Intermediario y de Autogestión. Naranja y mandarina se comercializan en un $80 \%$ en el Mercado Presencial. Para el caso de El Colegio existe una tendencia clara a comercializar a través de los Mercados Presenciales, a diferencia de lo que sucede en el municipio de Fuente de Oro. La posible causa de esto es que los productores buscan el espacio donde mejores ganancias obtengan, y para este caso es la vía de Mercados Presenciales. Los tiempos dedicados a cada vía de comercialización van desde 12 horas en Mercados Presenciales, hasta 2 horas a través del Mercado Intermediario. A diferencia del caso anterior, en el Mercado Intermediario es donde se recibe el pago más rápidamente. En ocasiones, en los Mercados Mayoristas, el pago sucede luego de dos o tres días. Sin embargo, existe una desventaja en el Mercado Intermediario ya que el pago por los productos resulta ser inferior al recibido por otras vías. La Tabla 4 muestra la utilidad neta por una arroba de producto, donde se evidencia que los mejores ingresos se siguen recibiendo por la vía de Mercados Presenciales.

\begin{tabular}{lccc}
\hline & Mercados Presenciales & Mercados Mayoristas & Mercado Intermediario \\
\hline Banano primera & 8200 & 1200 & 3200 \\
Banano segunda & 6200 & -800 & 1200 \\
Banano bocadillo & & & \\
Mandarina primera & -1800 & -800 & 1200 \\
Mandarina segunda & 17000 & -8000 & -8000 \\
Naranja & -7000 & -10000 & -10000 \\
Limón & -13000 & -15000 & -15000 \\
\hline
\end{tabular}


Tabla 4. Utilidad neta en pesos Colombianos por arroba de producto. Caso El Colegio.

Los resultados evidencian que la mejor utilidad neta se recibe en el canal de los Mercados Campesinos por la vía del Mercado Presencial. Sin embargo, se evidencia que el productor en algunos productos está obteniendo pérdidas en las tres vías de comercialización. De ellas, es en el Mercado Presencial donde las pérdidas son menores. A pesar de esta situación, para el productor existe un alto grado de satisfacción, argumentando que en Mercados Campesinos es una alternativa excelen- te de comercialización porque puede interactuar directamente con el consumidor y allí se valora mejor su trabajo y su producto.

\subsection{Comparación de los dos municipios}

Con base en los resultados anteriores, es importante hacer una comparación de los dos municipios analizados, ver tabla 5 .

\begin{tabular}{|c|c|c|}
\hline Caso & Fuente de Oro & El Colegio \\
\hline Cultivos principales & Plátano, Yuca, Limón & $\begin{array}{l}\text { Mandarina, Naranja, Mango, Agua- } \\
\text { cate }\end{array}$ \\
\hline Cultivos secundarios & $\begin{array}{l}\text { Zapote, Guanábana, mango, Naranja, Ca- } \\
\text { cao, Aguacate, Noni }\end{array}$ & Banano, Plátano, \\
\hline Canales de comercialización & $\begin{array}{l}\text { Mercados Campesinos y Mercados Tradi- } \\
\text { cionales }\end{array}$ & $\begin{array}{l}\text { Mercados Campesinos y Mercados } \\
\text { Tradicionales }\end{array}$ \\
\hline Vías de comercialización & $\begin{array}{l}\text { Mercados Presenciales, Mercados Mayo- } \\
\text { ristas, Mercados de Autogestión }\end{array}$ & $\begin{array}{l}\text { Mercados Presenciales, Mercados } \\
\text { Mayoristas, Mercado Intermediario }\end{array}$ \\
\hline Mercados Presenciales en Bogotá & Parque el Olaya & Parque Kennedy, Policarpa \\
\hline Mercados Mayoristas & $\begin{array}{l}\text { Fruver y Plazas de Mercados Comité co- } \\
\text { mercialización }\end{array}$ & $\begin{array}{l}\text { Comité Comercialización Mercados } \\
\text { Campesinos }\end{array}$ \\
\hline Mercados Autogestión & $\begin{array}{l}\text { Plazas locales, Granada, San Martín, Fuen- } \\
\text { te De Oro }\end{array}$ & No participa \\
\hline Mercados Intermediarios & No participa & Intermediarios locales \\
\hline Vía de menores ingresos & Mercados Intermediario, por eso no lo usa & Mercados Intermediarios \\
\hline Vía de mayores ingresos & Mercados Presenciales & Mercados Presenciales \\
\hline Grado de satisfacción & 5 & 4 \\
\hline
\end{tabular}

Tabla 5. Comparación de los casos Fuente de Oro y El Colegio.

El elemento más importante a discutir en el final de este análisis es que, con base en la comparación de los casos analizados, la vía de comercialización en la que reciben menores ingresos es Mercados Intermediarios. En contraste, la vía en la que reciben una mejor remuneración es Mercados Presenciales. Es importante mencionar que incluso para los Mercados Presenciales, en el caso de El Colegio, el productor pierde dinero para el banano bocadillo, mandarina de segunda clase, y naranja.
Por el contrario, el caso de Fuente de Oro muestra resultados positivos para todos sus productos por la Vía de Mercados Presenciales. En ambos casos el grado de satisfacción que sienten los productores usando éstas Vías de Comercialización es positivo, alcanzando los grados 4 y 5 .

Estos resultados se pueden explicar en un hecho fundamental, el reconocimiento social y valor que el productor siente por su trabajo en los Merca- 
dos Presenciales. En Madison, Wisconsin (Estados Unidos) en el verano de 2014, durante el panel de discusión 'Voces de los sistemas alimentarios locales' llevada a cabo en la versión 78 de la reunión anual de la Sociedad de Sociología Rural de Norte América, Kriss Marion y Lori Stern, productores locales, presentaron sus ideas con relación al factor fundamental que a su juicio permitiría que los campesinos pudieran mejorar realmente su calidad de vida. Su conclusión fue que todo aspecto relacionado con la justicia social, es fundamental. Según ellos, esta justicia social tomaría cuerpo al momento de contar con unas políticas públicas que reconocieran la importancia del campesinado para toda la sociedad (Pachón, Bokelmann, \& Ramirez, 2016). Precisamente ese reconocimiento social es el que estarían calificando los productores de los casos estudiados, en la medida en que existe una comunicación directa con los consumidores, y éstos últimos evidencian la importancia que tiene el campesinado productor de alimentos para sus vidas diarias. El hecho de conocer a quien produce la comida, entender su realidad y su racionalidad, definitivamente permite otorgar un real valor al consumo de alimentos, aspecto que sin duda trasciende la transacción comercial (Parrado, \& Gutierrez, 2014). En otras palabras, no es lo mismo comprar alimentos en un almacén de grandes superficies, que adquirirlo en un Mercado Presencial de la mano directa de quien lo produce.

Aparte de lo anterior, Parrado y Molina (2014) destacan otro aspecto de gran importancia, evidenciado en los Mercados Presenciales, correspondiente a la capacidad de trueque que, una vez finalizado el mercado, se lleva a cabo entre los mismos productores. Aquellos que provenían de climas fríos, intercambiaban los productos que no habían sido comercializados con quienes provenían de otro tipo de climas. Sin lugar a dudas este intercambio comercial sin intermediación de dinero permitió que todos se vieran beneficiados, y que quienes participaban del trueque, adquirieran alimentos que no se producen en sus predios.
De igual manera, llama la atención que la intermediación, en al menos uno de los casos, no fuera utilizada con regularidad. Sin embargo, durante la investigación se evidenció un aspecto destacado por Forero (2000), con relación al papel que juegan en algunos lugares los intermediarios, ya que en muchos casos dichos actores mantienen estrechas relaciones de amistad y compadrazgo con los productores, y se convierten en los que proveen transporte para insumos, materiales, y personas, en lugares donde el acceso a transporte público es inexistente.

Finalmente, vale la pena dejar un interrogante que ha sido parcialmente discutido en otros estudios, y planteado por Melo (2015) en el sentido de cuestionarse sobre la sostenibilidad que procesos como el PMC podría tener en el tiempo. La realidad de este tipo de iniciativas es que sin un adecuado apoyo institucional, tenderían a desaparecer. Infortunadamente la idea de ayudar a la agricultura y al campesinado en una era de políticas neoliberales y en donde los gobiernos cada vez buscan desentenderse más de este tipo de asuntos, dista de llegar a convertirse en una realidad. Sin embargo, cada vez adquiere una mayor relevancia el hecho de que la agricultura requiere soporte gubernamental para su sobrevivencia. Dicho soporte necesariamente debe ir más allá de subsidios a los productores. Este apoyo debe tomar cuerpo en aspectos en los cuales Colombia tiene un gran rezago: vías de comunicación, carreteras, acceso a educación, salud, insumos, capacitación, entre muchos otros. Los mencionados aspectos hacen parte de la justicia social mencionada anteriormente, y que el campesinado en Colombia, liderado por los campesinos Boyacenses, ha puesto de manifiesto en los últimos paros agrarios.

\section{Conclusiones}

Los mercados campesinos por la vía Mercados Presenciales es el canal por el cual los productores, para este estudio, obtienen mayores ingresos por arroba de producto comercializado. Es importante destacar que los productores tienen un mayor 
agrado por los Mercados Presenciales, dado que sienten una afinidad con los consumidores y reciben mejores ingresos, aunque no descartan las otras vías. Esta vía de comercialización cara a cara facilita un proceso en el que existe un diálogo entre productores y consumidores, llegando a establecer relaciones diferentes que cuando se compra a un intermediario o en un Mercado Mayorista o supermercado.

El trasporte es un elemento fundamental durante el proceso de comercialización. Cuando hay poca disponibilidad, la dificultad y costos se incrementan. Esto ha implicado que muchas veces los productores no puedan sacar sus productos del predio, y por tanto no lo puedan vender.

\section{Agradecimientos}

Los autores agradecen al Comité de Interlocución Campesina, CIC, y a sus organizaciones; al Programa Mercados Campesinos, a la Alcaldía Mayor de Bogotá a través de la Secretaría de Desarrollo Económico; al Oxford Committee for Famine Relief, OXFAM, y al Instituto Latinoamericano para una Sociedad y un Derecho Alternativos, ILSA, por facilitar las condiciones para la realización de esta investigación. Especial agradecimiento a José, Rosalba e hijos, así como a Fabio López y Alirio Gómez, por facilitar un espacio para estudiar su diario quehacer.

\section{Referencias}

Bartra, A. (1982). El comportamiento económico de la producción campesina. Texcoco, Mexico: Universidad Autónoma de Chapingo.

Benítez, J. A. (2009). La economía campesina y la comercialización de productos agropecuarios. Entramado, 4 (1), 3-10.

Bernstein, H. (2010). Class dynamics of agrarian change 1. Winnipeg: Kumarian Press.
Chaparro, A. M. (2014). Sostenibilidad de los sistemas de producción campesina en el proceso mercados campesinos (Colombia). España: Universidad de Córdoba.

Chayanov, A. V. (1986). AV Chayanov on the theory of peasant economy. Manchester University Press.

Forero, J. (2000). Economía campesina y sistema alimentario en Colombia: Aportes para la discusión sobre seguridad alimentaria. Facultad de Estudios Ambientales y Rurales, Universidad Javeriana. Recuperado de: http://www.javeriana.edu.co/ear/d_ des_rur/documents/campesinadoysistemaalimentarioencolombia.pdf

Forero, J. (2003). Economía campesina y sistema agroalimentario en Colombia: aportes para la discusión sobre seguridad alimentaria. Economía Campesina Y Sistema Alimentario En Colombia: Aportes Para La Discusión Sobre Seguridad Alimentaria, 44.

Gonzalves, G. (2007). Economía campesina y economía comunitaria: Apuntes para analizar las experiencias en proyectos de desarrollo rural. Santa Cruz, Bolivia.

ILSA, Campesina, C. de I., \& OXFAM. (2010). Resultados, lecciones aprendidas, y desafíos, 2007-2010. Bogotá.

Machado, A. (2003). La cuestión agraria frente al neoliberalismo. La Falacia Neoliberal. Crítica Y Alternativas, 269-282.

Melo, F. (2015). Análisis de sostenibilidad del proceso de Mercados Campesinos de la Región Central de Colombia. In V Congreso Latinoamericano de Agroecología-SOCLA. La Plata, Argentina.

Pachón-Ariza, F., Bokelmann, W., \& Ramirez, C. (2016). Heritages of the peasantry: an analytical framework to address rural development. In 4th 
Biannual Conference on Nordic Rural Research. Akureyri, Iceland: Akureyri University.

Parrado, A., \& Gutierrez, O. (2014). Investigando con campesinos. La experiencia de acompañamiento al proceso mercados campesinos. En F. Pachon \& J. Molina (Eds.), Investigando y actuando en territorios rurales 132-155. Bogotá: Editorial Universidad Nacional de Colombia.

Parrado-Barbosa, A., \& Molina, J. (2014). Mercados campesinos: modelo de acceso a mercados y seguridad alimentaria en la región central de Colombia. Colombia: Oxfam.

Pitre-Redondo, R., Cardona-Arbeláez, D., \& Hernández-Palma, H. (2017). Proyección del emprendimiento indígena como mecanismo de competi- tividad en el postconflicto colombiano. Revista de Investigación, Desarrollo e Innovación, 7 (2), 231240. doi: https://doi.org/10.19053/20278306. v7.n2.2017.6068

Plaza, O. (1992). Economía campesina: límites y potencialidades en el contexto de apertura y modernización. Seminario Internacional: Desarrollo Rural Y Apertura Económica. Santafé de Bogotá, Colombia. Fondo DRI-IICA.

Sierra-Roberto, J. D. F. (2012). Perspectivas y escenarios de desarrollo rural para las comunidades campesinas: estudio de caso Municipio de Sora, Boyacá, Colombia. Revista de Investigación, Desarrollo e Innovación, 2 (2), 8-17. Recuperado de: http://revistas.uptc.edu.co/revistas/index.php/investigacion_duitama/article/view/1311 